\title{
Importance of biodiversity to the modern pharmaceutical industry*
}

\author{
Robert N. Young \\ Merck Frosst Centre for Therapeutic Research, PO Box 1005, Pointe Claire - Dorval, \\ Québec, Canada, H9R 4P8
}

\begin{abstract}
Natural substances have long served as sources of therapeutic drugs. Many substances have been derived from traditional medicine, e.g. digitalis (from Foxglove), ergotamine (from contaminated rye), quinine (from Cinchona), etc. More recently, many antibiotic, antifungal and anticancer agents have been derived from bacteria, fungi, plant and animal sources. While newer techniques of combinatorial chemistry and large scale screening of synthetic products as well as de novo design are now a mainstay for new drug discovery, the search for new natural products continues as an importance source of structural diversity. Genomic research continues to identify molecular targets for disease which can derive specific screening assays. All major drug companies screen plant, bacterial and fungal extracts, as well as synthetics. However, rarely, is a screening active discovered ideally suited for marketing as a drug due to suboptimal bioavailability, half-life, toxicity, specificity, etc. More often, natural products provide lead structures which are starting points for chemical modification to derive an optimal drug.

Many new companies have been set up in the last few years to exploit natural products. As well, new techniques of combinatorial biosynthesis and gene transfer offer possibilities for identification of novel substances heretofore unaccessible for testing. It is critical that biomass diversity be maintained to provide future structural diversity and provide leads and drugs for pharmaceutical targets that will emerge in the coming years.
\end{abstract}

\section{INTRODUCTION}

The preservation of biodiversity throughout the world is of importance to the human population and indeed to the stability of the entire world. The vast genetic variety available in terrestrial plants, animals and micro-organisms offers a wealth of possibilities for the betterment of mankind in the production of food, materials and medicine. It is the intention of this paper to present a personal view of the importance of biodiversity to the modern pharmaceutical industry with particular reference to examples available from Merck Research Limited.

For modern pharmaceutical research, biodiversity equals chemical diversity. Natural products offer a vast source of chemical diversity and yield unusual and unexpected lead structures. Although not always understood, secondary metabolites often have important biological function and are generally produced by plants, animals and micro-organisms for specific reasons. As such, small molecules produced in this way have been 'designed' by nature to interact with macromolecules (proteins, DNA, etc.) and thus modulate the function of such macromolecules. Natural products can derive not only lead structures but can often yield ready-made drugs.

\footnotetext{
* Plenary lecture presented at the 2nd International Conference on Biodiversity, Belo Horizonte, Brazil, 11-15 July 1999, pp. 1611-1690.

Correspondence: E-mail: ryoung@ @erck.com
} 


\section{HISTORICAL PERSPECTIVE}

Drug discovery has evolved over many centuries. Historically, teas and extracts were derived from natural sources and through trial and error found to have some efficacy to treat human diseases. Such experiences were collected by shamen and healers, passed on from generation to generation, building up small but important armaments of treatment. Virtually all indigenous populations have derived a pharmacopoeia which is unique to their environment. In the last two centuries, as chemistry developed, pure substances and synthetic analogs became available. Potent and effective plant, animal or bacterial extracts were purified to derive active ingredients which generally had better properties than the crude extracts. As well, animal models of diseases were developed which allowed testing of these substances and derived analogs with the hopes of minimizing toxicity and optimizing potency. However, due to the complexity of the models, optimizations were generally slow and difficult. In the latter half of the twentieth century, advances in cell biology and biochemistry allowed the setting up of in vitro testing and screening using animal and, in some cases, human tissues and cell models. It then became possible to optimize medicinal substances before the more complicated and ambiguous testing in animals and humans. This led to a greater emphasis on lead optimization and the derivation of more specific drugs which had minimized side-effects and ancillary activities.

The last two decades have been characterized by what could only be called the molecular biology revolution. The cracking of the genetic code and the derivation of methodologies to characterize the genome have led to a ready availability of a vast array of proteins, either in pure states or as expressed in modified cells. This has led to the ability to characterize receptors and enzymes, both for their function and for their structural biology utilizing advanced techniques such as X-ray and NMR spectroscopy. The identification of macromolecule targets for drug development and their involvement in disease has allowed genetic typing and the correlation of genetic defects with certain types of diseases which has further allowed us to pinpoint important new biochemical targets for disease treatments. We now have a relatively facile ability to knock out specific proteins in animals or in cells and then observe the consequences of the lack of such a protein and its correlation with disease or possible treatment. These powerful techniques are leading to the discovery of a myriad of potential drug targets which represents a huge potential for the pharmaceutical industry to discover novel and specific therapies for the betterment of mankind. Similarly, the understanding of genetics is leading to the development of a molecular basis for toxicity, metabolism, absorption and all the other pharmaceutical properties important in designing optimal drug substances. Other technological advances have led to the development of a variety of tools to rapidly produce and test many compounds. High throughput screening and combinatorial chemistry are now going hand-in-hand to more rapidly advance the discovery of novel and specific chemical entities. Similar techniques can be used to rapidly test a wide variety of naturally derived substances, either in a pure or impure state. All this has led to a voracious appetite of the pharmaceutical industry for a vast number of structures to test. It must be emphasized that as these new targets are defined, it is important to re-screen all available samples, even if they haven't shown interesting activity in the past. New assays and new specific targets offer new opportunities for old compounds.

There is a wide variety of naturally derived drugs which still serve as important therapies in medicine today. Many also have served as tools to pinpoint mechanisms of action and to better understand the basis of physiology and disease. Examples of drugs which have been utilized for many years and still have importance are digitalis for heart disease; Aspirin (derived from salicilin) for pain and inflammation; quinine for treatment of malaria; atropine and scopolamine (derived from Atropa belladonna) and used as anti-cholinergic agents and as anaesthetic; morphine (derived from poppy) and still used as a potent analgesic; ergot alkoloids, ergotamine and ergonovine (derived from Claviceps purpurea, a fungus parasitic on grain), and utilized to treat migraine headaches and to accelerate birth or as an abortioninducing agent; ephedrine (derived from Ephedra sinica and used in the Far East for more than two millenia) for treatment of asthma and colds and still used in OTC medications; reserpine (derived from Rauwolfia serpentina) and used as an anti-psychotic by herbalists in India before 1000 B.C. Reserpine itself was used, until recently, as an anti-psychotic and it is still used for hypertension and in Reynaud's syndrome; pilocarpine (derived from Pilocarpus microphyllus), a rain forest shrub native to Brazil. It was 
long used by Amazonian tribes to treat mouth ulcers, colds and flus and it is still used to lower interocular pressure to treat glaucoma.

Some more recent examples include the anti-cancer agents, vinblastine and vincristine from the common periwinkle, which is used to treat Hodgkin's Disease, leukemia and testicular cancer; Etoposide and Podofilox isolated from Mayapple and used to treat testicular cancer, small cell lung cancer and genital warts; Taxol which is obtained from western yew and used to treat ovarian and lung cancer.

\section{NATURAL PRODUCTS AND DRUG DISCOVERY TODAY}

Thus, natural products remain as important sources of new drugs. Statistics show that in the 10 years from 1983 to 1994, while only about 5\% of newly approved drugs were natural products, about $35 \%$ were either compounds derived directly from natural products or synthetics based on natural products [1]. Because of the importance of natural products as leads or as drugs in themselves, most major pharmaceutical companies maintain important efforts in natural products' research. Modern techniques of rapid screening and purification have enhanced such efforts and natural organisms are recognized as important sources of a wide variety of chemical classes and novel structures. Indeed, natural products could be considered as privileged structures, which due to their putative interaction with macromolecules, should be considered to have far better odds to show biological activity relative to random synthetic compounds. It is rare today that natural products are developed as such and generally must be modified to improve pharmaceutical and biological properties or to reduce toxicity.

In recent years, pharmaceutical natural products' research has tended to favor compounds derived from bacteria and fungi because of their diversity of species and ease of culture and because if active compounds are discovered, generally it is possible to culture and manipulate micro-organisms to enhance the production of the wanted compounds. Complex products derived from plants and animals may prove more difficult due to the rarity of the species and difficulty in cultivation or collecting raw materials.

Merck has developed many natural products over the years including Zocor $^{\mathrm{TM}}$, Mevacor ${ }^{\mathrm{TM}}$, Primaxin $^{\mathrm{TM}}$, Ivermectin ${ }^{\mathrm{TM}}$ and Mefoxin ${ }^{\mathrm{TM}}$ to name but a few that are currently on the market. These have all been derived from micro-organisms. Microbiological diversity is enormous and has only partially been investigated. It has been estimated that only 5000 bacteria have been identified out of a possible $>40000$ total and about 70000 fungi are known of what may be 1500000 total. Microbiological samples are obtained from a variety of sources including soil, dung, leaf litter, on plants, animals, insects and water samples. A variety of techniques may be used to isolate micro-organisms from such mixtures including using selective media, special treatments, i.e. heat and growing up organisms to collect specialized fruiting bodies or mycilia. Special fermentation techniques may favor production by unique organisms or enhance production of particular metabolites. Special media or stress conditions can favor production of unique metabolites or enhance the survival of particular organisms.

Merck has set up a variety of collaborations as well as in-house efforts to collect natural products, both plant and micro-organisms and to test them in rapid high throughput screening. Merck maintains a significant natural products department in its Rahway, New Jersey laboratories and an important testing facility in Madrid, Spain, where high throughput screenings are run on many thousands of extracts per year. Some interesting compounds isolated through such screening by Merck in recent years include actinoplanic acids A \& B (1a, b) which were identified from an Actinoplanes species isolated from lichen on an oak tree in Spain and shown to be potent inhibitors of farnesyl-protein transferase, an enzyme essential for membrane association of the ras ocogene and required for cell transformation in cancer [2,3]. Merck's screening for anti-protozolal agents using a culture assay with Eimeria species led to the discovery of Apicidin (2), a broad spectrum anti-protozoal agent which was isolated from Fusarium pallidoroseum collected from branches of an Acacia tree is Costa Rica. [4]

Plant samples have also derived interesting structures which have been used as leads for Merck research. One example is correolide (3), a triterpene derived from Spachea correae collected in Costa Rica and shown to be a potent reversible blocker of a potassium channel important in T-cell receptor activation [5]. Due to the complexity of the structure and its less than optimal pharmaceutical properties, it could not be used as such. It was, however, studied extensively in order to try and derive a more simple and tractable pharmacaphor [6]. Unfortunately, these efforts led to only limited success. Another 
interesting lead structure from a plant source, justicidin E (4), isolated from Justica procumbens by Stevenson and Holmes [7], was shown to be a potent inhibitor of 5-lipoxygenase, an important enzyme in the biosynthesis of leukotrienes. Such inhibitors have shown efficacy in the treatment of human asthma. Justicidin E served as a lead structure in our laboratories to develop a number of modified lignan-like compounds which showed nanomolar activity and good bioavailability and oral activity. One such compound, L-739,010 [8] (5), reached early safety assessment but, unfortunately, development was stopped due to toxicity which was apparently associated with the formation of reactive metabolites $[9,10]$.

Many natural products have served as sources for synthetic medicinal chemistry drug discovery and as receptor and enzyme targets have become more available through cloning and expression systems, many improved drugs have been obtained from less optimal natural products. An excellent example is the evolution of seratonin $(5-\mathrm{HT})$ receptor agonists and antagonists for treatment of migraine headaches. The natural product, ergotamine (6), was marketed by Sandoz. We now understand that this compound is relatively nonspecific and interacts with a number of 5 -HT receptors including $1 \mathrm{~A}, 1 \mathrm{~B}, 1 \mathrm{D}$ and $1 \mathrm{~F}$. Due to its vaso-constrictor activity, it aborts migraine headache but also is associated with a variety of CNS sideeffects. Sandoz later introduced methysergide (7) with a somewhat simplied structure but still associated with hallucinations and CNS side-effects. In 1991, Burroughs Wellcome introduced sumatriptan (8), a more specific 5-HT1B/1D agonist, with much less side-effects than ergotamine or methysergide. Most recently, Merck has introduced rizatriptan $\left(\right.$ Maxalt $^{\mathrm{TM}}$ ) (9) (in 1998), a 5-HT 1B/1D specific agonist, with an improved side effect profile and a fast onset of action. Thus, ergotamine allowed modern research to understand its mode of action, to identify these receptors as an important target and to develop improved drugs (Scheme 1).

Most complex natural products lack the optimal pharmaceutical properties necessary to be viable in the current modern drug environment. Merck research has recently identified an important anti-fungal compound, known as caspofungin (10) which was derived from a complex polipeptide anti-fungal compound, pneumocandin $\mathrm{B}_{0}(\mathbf{1 1})$ which was discovered in 1985 from a fungus, Glarea lozoyensis. This compound showed good anti-fungal activity for Candida although poor activity on Aspergillus species. It also had poor oral bioavailability and very low water solubility. Extensive studies in the Merck Laboratories on pneumocandins and related echinocandins led to the synthesis of more soluble analogs through the appending of basic groups to the natural product. Compounds such as L-733560 (12) had improved potency, stability and water solubility [11]. Final optimization derived the tri-basic analog, caspofungin (or Cancidas ${ }^{\mathrm{TM}}$ ). The compound is currently in advanced clinical studies and has thus far shown efficacy in a variety of eosophagal and oropharyngal fungal infections (Scheme 2).

So it can be seen that natural products can be anticipated to continue to contribute to Merck's drug discovery efforts for the forseeable future.

\section{THE FUTURE}

In the future, natural products will remain as important sources of new drugs and lead structures. With improving techniques of organic synthesis, plant fungal and bacterial culture and bioengineering, more and more complex substances will become readily available for testing, degradation or modification. Varieties of new techniques will enhance the discovery process and allow identification of active substances from complex mixtures. Newly evolving techniques such as fishing techniques, wherein extracts from complex mixtures are absorbed on affinity matrixes, including the target macromolecules, and then subsequently eluted off and identified by mass spectral and NMR techniques, will allow the identification of active substances in a more rapid and efficient fashion.

It is clear that many plants and micro-organisms have not yet been studied and can serve as a vast source of potential lead substances. Many companies have been formed in the last decade to exploit natural products. Some such as Shaman Pharmaceuticals, Phytobiotech and Microbotanica, etc. have focused on natural products from tropical and other medicinal plants identified by native cultures around the world. Others such as PharmuMar, Aquaculture Technology and ChromaXome focus on natural products from marine organisms.

Some of the most exciting and futuristic opportunities are being explored by companies applying new molecular biology techniques to find novel, natural and unnatural products. Companies such as Ecopharm 
<smiles>CCC(CC(C)C(=O)O)C(=O)CCC(CO)CC(C)CC(C)=CC(C)C(O)C(CCC(CC(=O)O)OC(=O)CC(CC(=O)O)CC(=O)O)CC(=O)OC(C)CCC(C)CCCCCCC(=O)O</smiles>

actinoplanic acids $A$ and $B$ (lactone)(1a,b)

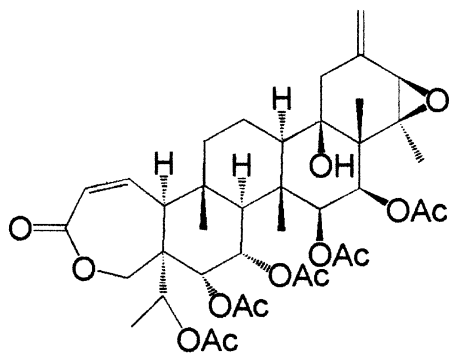

correolide (3)<smiles>O=C1OCc2c1cc1c(-c3ccc4c(c3)OCO4)c2C=C2OCOC2=C1</smiles>

justicidin E (4)<smiles>CCCCN(CCC)CC(=O)NC(CCCCC[C@H](O)CC)C(=O)NC(Cc1cn(OC)c2ccccc12)C(=O)NC(C(C)O)C(C)CC</smiles>

apicidin (2)

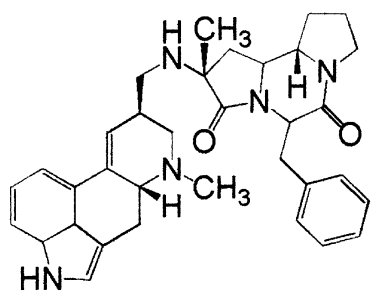

ergotamine (6)<smiles>CCC(CO)NC(=O)[C@@H]1C=C2C3=CC=CC4NC=C(CC2N1)C34</smiles>

methysergide (7)<smiles>C=NS(=O)(=O)c1ccc2[nH]cc(CCN(C)C)c2c1</smiles>

sumatriptan (8)<smiles>N#Cc1cc(-c2ccoc2)c2ccc(COc3cccc(C4(O)CC5COC(C4)O5)n3)cc2c1</smiles>

L-739,010 (5)<smiles>CN(C)CCc1c[nH]c2ccc(Cn3cncn3)cc12</smiles>

rizartiptan (9)

Scheme 1

concentrate on finding compounds from micro-organisms associated with plants that are resistant to insects, fungi or other infections. Several other companies, such as Ecopia Biosciences, Diversa, Microgenomics, Ambergene and Terragen Diversity are applying cloning techniques in attempts to sample so-called 'uncultivatable micro-organisms' (which may represent 95-99\% of existing micro-organisms!) [12].

Some companies such as Ariad, Diversa and Oceanix have reported on cloning techniques applied to such purposes. They have developed particular vectors which allow the incorporation of large fragments of DNA derived from unculturable soil bacteria into readily cultured hosts such as E. coli. It is hoped that these large DNA fragments (up to $300 \mathrm{~kb}$ ) would include the necessary genes coding for particular small molecule biosynthesis and once cultured in the host bacteria, would derive interesting natural products which could be extracted and tested. The success of such techniques is yet to be demonstrated.

Finally, a company, Kosan, is working to utilize a technique known as combinatorial biosynthesis. 


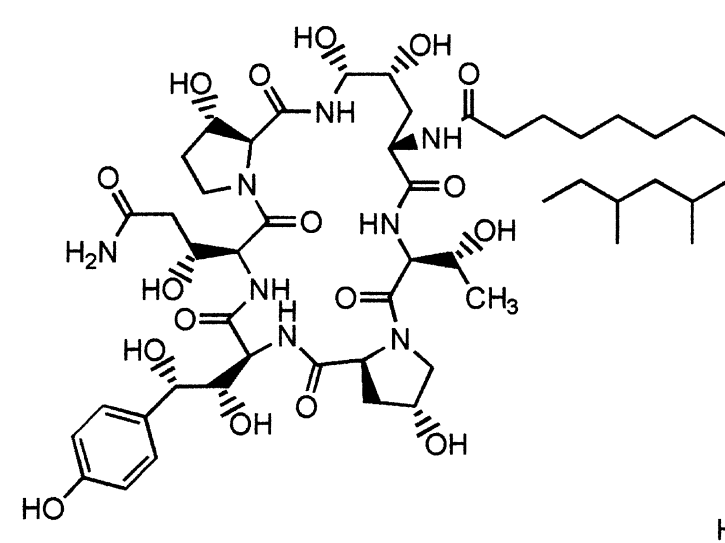

pneumocandin $\mathbf{B}_{0}(11)$

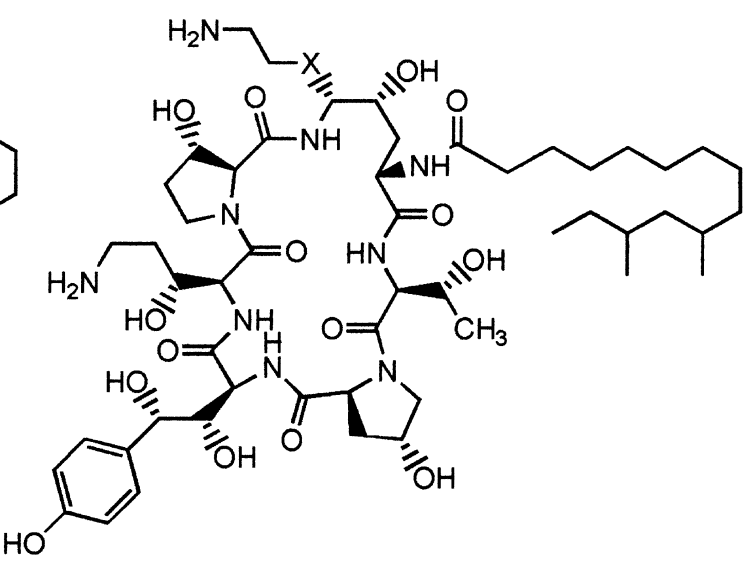

$X=0 ;$ L-733,560 (12)

$\mathrm{X}=\mathrm{NH}$; caspofungin, $\left(\right.$ Cancidas $\left.^{\mathrm{TM}}\right)(10)$

\section{Scheme 2}

Polyketide biosynthesis (involved in the biosynthesis of a wide variety of phenolic and ketonic natural products) makes use of large multifunctional enzymes made up of repeating cassettes. The sequence of cassettes dictates the structure, substitution and oxidation state of the final product. They hope to use DNA manipulation techniques to add, subtract, or swap cassettes in random combinatorial fashion and therefore make large libraries of 'unnatural natural products'. High throughput microscreening of such libraries would then hopefully identify interesting active compounds and the clones could be grown up and overexpressed in order to identify the active constituent.

It is clear that as purification, identification and testing technology improve, more and more unique natural products will become of interest to the pharmaceutical industry. Even rare and difficulty available samples may derive interesting compounds and, with these techniques, structures can be determined. Advances in synthetic chemistry can now make available complex structures heretofore beyond easy reach. There is little doubt therefore that natural products will continue to have an important impact on the pharmaceutical discovery process and it is equally imperative that we all work to preserve the genetic diversity of the world of living species on this earth as a resource for future generations.

\section{REFERENCES}

1 G. M. Cragg, D. J. Newman, K. M. Snader. J. Nat. Prod. 60, 52 (1997).

2 R. S. Iyer, B. F. Coles, K. D. Raney, R. Their, F. P. Guengerich, T. M. Harris. J. Am. Chem. Soc. 116, 1603 (1994).

3 S. B. Singh, J. M. Liesh, R. B. Lingham, K. C. Silverman, J. M. Sigmund, M. A. Goetz. J. Org. Chem. 60, 7896 (1995).

4 S. B. Singh, D. L. Zink, J. D. Polishook, A. W. Dombrowski, S. J. Darkin-Rathray, D. M. Schmatz, M. A. Goetz. Tetrahedron Lett. 37, 8077 (1996).

5 M. A. Goetz, O. D. Hensens, D. L. Zink, R. P. Borris, F. Morales, G. Tamayo-Castillo, R. S. Slaughter, J. Felix, R. G. Ball. Tetrahedron Lett. 39, 2895 (1998).

6 M. MacLoss, M. Goetz, G. Kaczorowski. Unpublished results.

7 T. L. Holmes, R. Stevenson. J. Org. Chem. 36, 3450 (1971).

8 P. Hamel, D. Riendeau, C. Brideau, C.-C. Chan, S. Desmarais, D. Delorme, D. Dubé, Y. Ducharme, D. Ethier, E. Grimm, J. P. Falgueyret, J. Guay, T. R. Jones, E. Kwong, M. McAuliffe, C. S. McFarlane, H. Piechuta, M. Roumi, P. Tagari, R. N. Young, Y. Girard. J. Med. Chem. 40, 2866 (1997).

9 K. E. Zhang, J. A. Naue, B. Arison, K. P. Vyas. Chem. Res. Toxicol. 9, 547 (1996). 
10 N. Chauret, D. Nicoll-Griffith, R. Friesen, C. Li, L. Trimble, D. Dubé, R. Fortin, Y. Girard, Yergey. J. Drug Metab. Dispos. 23, 1325 (1995).

11 F. A. Bouffard, R. A. Zambias, J. F. Dropinski, J. M. Balkovec, M. L. Hammond, G. K. Abruzzo, K. F. Bartizal, J. A. Marrinan, M. B. Kurtz, D. C. McFadden, K. H. Nollstadt, M. A. Powles, D. M. Schmatz. J. Med. Chem. 37, 222 (1994).

12 A. M. Rouhi. Chem. Eng. News 77, 21 (1999). 